\title{
Self-Organized Pacemakers in a Coupled Reaction-Diffusion-Mechanics System
}

\author{
A. V. Panfilov and R. H. Keldermann \\ Theoretical Biology, Utrecht University, Padualaan 8, Utrecht, 3584 CH, The Netherlands
}

M. P. Nash

Bioengineering Institute and Department of Engineering Science, The University of Auckland, Private Bag 92019, Auckland, New Zealand

(Received 27 January 2005; published 15 December 2005)

\begin{abstract}
Using a computational model of a coupled reaction-diffusion-mechanics system, we find that mechanical deformation can induce automatic pacemaking activity. Pacemaking is shown to occur after a single electrical or mechanical stimulus in an otherwise nonoscillatory medium. We study the mechanisms underpinning this effect and conditions for its existence. We show that self-organized pacemakers drift throughout the medium to approach attractors with locations that depend on the size of the medium, and on the location of the initial stimulus.
\end{abstract}

DOI: 10.1103/PhysRevLett.95.258104

Reaction-diffusion equations describe a wide variety of nonlinear systems in physics, chemistry, and biology. They describe nonlinear wave patterns in the BelousovZhabotinsky (BZ) chemical reaction [1], in the processes of morphogenesis of the mould Dictyostelium discoideum (Dd) [2], of electrical activity in cardiac tissue [3], and in many other biological systems. Propagation of nonlinear waves is usually accompanied by other important processes. One of the most fundamental is the mechanical deformation of the medium. Indeed, the primary physiological function of the electrical waves in the heart is the initiation of cardiac contraction. Contraction of the heart in turn affects the process of wave propagation resulting in a complex global feedback phenomenon known as mechanoelectrical feedback, which has been studied in electrophysiology for well over a century [4]. Nonlinear waves during Dd morphogenesis induce motion of the cells, which substantially affects wave dynamics [2]. Waves in the $\mathrm{BZ}$ reaction in gels cause deformation [5], which in turn affects the spiral wave dynamics [6]. Furthermore, a "chain reaction" of spiral wave births and deaths can result from an externally controlled deformation of a medium [7]. Although the interplay of mechanical deformations with the dynamics of reaction-diffusion systems is an important phenomenon, most studies have separately considered the mechanical deformation or nonlinear wave propagation in reaction-diffusion systems.

Recently, we introduced a general framework to study the effects of mechanical deformation on reactiondiffusion systems [8]. We described a deformable, excitable medium capable of conducting nonlinear waves of excitation, which initiate contraction. The reactiondiffusion system was defined using a general curvilinear coordinate system, with a metric determined using the equations of continuum mechanics. In turn, deformations were initiated and controlled by the reaction-diffusion system. We illustrated this concept using simple reactiondiffusion models of cardiac excitation that have been suc-
PACS numbers: 87.19.Hh, 82.40.Np, 87.19.Nn, 87.19.Rr

cessfully applied in electrophysiology $[9,10]$. However, the formulation in [8] lacks several important feedback mechanisms, including a description of the stretch-activated transmembrane currents, which are considered to be the main physiological basis of mechanoelectrical feedback in cardiac tissue [4].

This Letter reports a novel phenomenon for coupled reaction-diffusion-mechanics systems: the formation of self-organized pacemakers. To this end, we have extended the electromechanical framework in [8] to include a description of the stretch-activated currents. We have investigated the mechanisms underpinning these self-organized pacemakers, and have studied their dynamical properties, such as drift patterns and the dependence of drift on the initial conditions, and medium size. Self-organization of pacemakers is important to the general theory of reactiondiffusion systems [11,12] as well as for applications such as pacemaker activity in cardiac tissue to initiate the normal heart beat [4].

Electromechanical model. - We start with the threevariable reaction-diffusion-mechanics model of excitable tissue introduced in [8]:

$$
\begin{gathered}
\frac{\partial u}{\partial t}=\nabla^{2} u-k u(u-a)(u-1)-u v-I_{s}, \\
\frac{\partial v}{\partial t}=\epsilon(u)(k u-v) \\
\frac{\partial T_{a}}{\partial t}=\epsilon(u)\left(k_{T} u-T_{a}\right) \\
\frac{\partial}{\partial X_{M}}\left(T^{M N} \frac{\partial x_{j}}{\partial X_{N}}\right)=0 \\
T^{M N}=\frac{1}{2}\left(\frac{\partial W}{\partial E_{M N}}+\frac{\partial W}{\partial E_{N M}}\right)+T_{a} C_{M N}^{-1} \\
\nabla^{2} u=\frac{\partial}{\partial X_{M}}\left(\sqrt{C} C_{M N}^{-1} \frac{\partial u}{\partial X_{N}}\right) .
\end{gathered}
$$


Equations (1) and (2) provide a standard lowdimensional model of cardiac electrical propagation: $u$ and $v$ are normalized variables representing the transmembrane potential and recovery properties of the tissue, respectively; $[-k u(u-a)(u-1)-u v]$ is the total transmembrane ionic current per unit area [8]. The parameters are $a$, which represents the threshold of activation (and is a key control parameter in the simulations); $k$, which controls the magnitude of transmembrane current $(k=8$ in all simulations); and $\epsilon(u)$, which sets the time scale of the recovery process: $\epsilon(u)=1$ for $u<0.05$, and $\epsilon(u)=0.1$ for $u \geq 0.05$. For the parameter values used in this Letter, Eqs. (1) and (2) describe nonoscillatory cardiac tissue that supports stable propagation of excitation waves.

The excitable tissue in our model contracts and the mechanics is modulated by the variable $T_{a}$ [described by Eq. (3)], which represents the active stress generated by the medium. $k_{T}$ governs the rate of tension development $\left(k_{T}=\right.$ 10 for all simulations).

Following standard continuum mechanics [13], we use two coordinate systems to define the deformation. $\left\{X_{M}\right\}$ are material coordinates embedded in the contracting tissue, and $\left\{x_{i}\right\}$ are the spatial reference Cartesian coordinates. The equations of continuum mechanics provide the relationship between these two coordinate systems $\left[x_{i}\left(X_{M}\right)\right]$; i.e., they determine the deformed position of a point $x_{i}$ that was initially located at $X_{M}$. This is achieved using the equations of stress equilibrium [Eq. (4)] that arise from the physical laws of motion (conservation of linear momentum). In this Letter, we use a stress tensor of finite elasticity theory, $T^{M N}$ [the second Piola-Kirchhoff stress tensor in Eq. (5)], which contains two parts: (i) the active stress components, $T_{a} C_{M N}^{-1}$, where $C_{M N}=\left(\partial x_{k} / \partial X_{M}\right)\left(\partial x_{k} / \partial X_{N}\right)$ is the right Cauchy-Green deformation (metric) tensor of the $\left\{X_{M}\right\}$ coordinate system [14], and (ii) the passive elastic stress components, which are expressed in terms of the derivatives of a strain energy function $(W)$ with respect to components of the Green's strain tensor, $E_{M N}=\frac{1}{2}\left(C_{M N}-\delta_{M N}\right)$, where $\delta_{M N}$ is the unitary tensor. For this study, the strain energy function was chosen to be the Mooney-Rivlin constitutive law [14], $W=c_{1}\left(I_{1}-3\right)+c_{2}\left(I_{2}-3\right)$, where $I_{1}$ and $I_{2}$ are principal invariants of $C_{M N}$, and $c_{1}$ and $c_{2}$ are stiffness coefficients, which together with the parameter $k_{T}$ from Eq. (3), determine local deformations during contraction $\left(c_{1}=2\right.$, $c_{2}=6$ and $k_{T}=10$ for all simulations, chosen to give rise to relative local deformations of approximately $15 \%$ following excitation, consistent with contracting cardiac tissue). Because of motion of the material coordinate system, we used a general curvilinear expression given by Eq. (6) to evaluate the Laplacian in Eq. (1), with $C=\operatorname{det}\left(C_{M N}\right)$, which provides a diffusive membrane current per unit undeformed area. Note that Eq. (6) differs from the equivalent expression in [8], for which the current was calculated per unit deformed area.

The direct influence of contraction on excitation is given by a stretch-activated current $I_{s}$, known to be present in cardiac tissue [4]. In this study, we have incorporated a generic description of the stretch-activated currents into the model using

$$
I_{s}=G_{s}(\sqrt{C}-1)\left(u-E_{s}\right),
$$

where $G_{s}$ and $E_{s}$ are the maximal conductance and reversal potential of the stretch-activated channels, respectively. This type of relation is similar to that used in [15], but in our model the deformation of the medium is computed as a part of the mechanics solution procedure, therefore we use an explicit dependence of the conductance on the local dilatation $(\sqrt{C}-1)$. Furthermore, since the current through the stretch-activated channels occurs mainly during stretch, we assume that the current in Eq. (7) is present only if $\sqrt{C}>1$. The value of the parameter $E_{s}$ in our model was typically 1 , and describes the depolarizing effect of the current observed experimentally $[4,15]$. The value of $G_{s}$ is one of the main determinants of the effects of deformation on wave propagation and was varied in our computations.

Numerical integration methods. - The coupled electromechanical model was solved using a hybrid approach that combines an explicit Euler time integration scheme to compute the excitation characteristics of the medium, with nonlinear finite element techniques to determine the large deformation mechanics of the tissue. The solution procedure is as follows: after $N_{\text {mech }}$ time integration steps for Eqs. (1)-(3), the equations governing tissue mechanics [Eq. (4)] are solved, subject to the active stresses $T_{a}$ due to Eq. (3). The solution of Eq. (4) provides updated values of the metric tensor components $C_{M N}$, which in turn modulate excitation properties [via Eqs. (6) and (7)] for the subsequent $N_{\text {mech }}$ excitation time steps.

The model solution parameters were the following: Euler computations were performed using a time integration step of $\Delta t=0.03$ (dimensionless time units) and a space integration step of $\Delta x=\Delta y=0.6$ (dimensionless space units), consistent with previous studies [8]. The mechanics mesh was defined using up to $15 \times 15$ finite elements. Each mechanical element contained up to $7 \times 7$ electrical grid points, and the value of $N_{\text {mech }}=3$ was used. Thus, the finite difference mesh was up to $91 \times 91$ grid points. These parameters were chosen following a solution convergence study to ensure that the main results of this Letter (i.e., the onset of pacemakers) were insensitive to these parameter choices. No-flux boundary conditions were imposed for Eq. (1), and the boundaries of the medium were fixed in space for Eq. (4). Mechanically, the fixed boundaries are consistent with an isometric contraction regime: a standard experimental procedure for muscle mechanics, during which end points of the tissue are fixed to maintain constant overall length. Full details of the mechanical and electrical coupling of the model, and the numerical (finite element) methods used to solve the equations of mechanics are given in [8].

Results. - The upper panels of Fig. 1 show the process of wave propagation after application of a single stimulus at 
the center of the medium, and in the absence of mechanical activity. We observed that the stimulus initiated a single wave of excitation [Fig. 1(a)] that vanished following propagation [Fig. 1(b)]. The spatiotemporal evolution of this pulse is presented in Fig. 1(c). In the presence of mechanical activity of the medium, we observed that the same initial conditions resulted in the onset of a stable pacemaker at the center of the medium [Figs. 1(d) and 1(e)]. Following a single stimulus, the initial propagating wave gave rise to subsequent waves that spontaneously appeared at the center of the medium [Fig. 1(f)]. Thus, mechanical deformation can initiate self-oscillatory activity in a reaction-diffusion system. The mechanism of this phenomenon is explained in Fig. 2(a), which illustrates the excitation variable $u$ (solid line) and local dilatation (dashed line) at the center of the medium during the pacemaking activity given in Figs. 1(d)-1(f). We observed contraction (negative dilatation) following excitation, but as the wave traveled away from the center, electrical recovery and localized stretch (positive dilatation) occurred in this region. This central stretch resulted from contraction of the surrounding parts of the medium and led to an inward current produced by the stretch-activated channels $I_{s}$ [Eq. (7)]. This current depolarized the central tissue resulting in the onset of a subsequent excitation.

The period of the pacemaker induced by the mechanical deformation was modulated by the parameter values. Increasing the stretch-activated current $I_{s}$ by increasing $G_{s}$ led to a decrease in the pacemaker period [Fig. 2(b)]. On the other hand, decreasing the excitation threshold $a$ also decreased the period of the pacemaker.

The parameters $G_{s}$ and $E_{s}$ that modulate the stretchactivated channels in Eq. (7) were both important for the
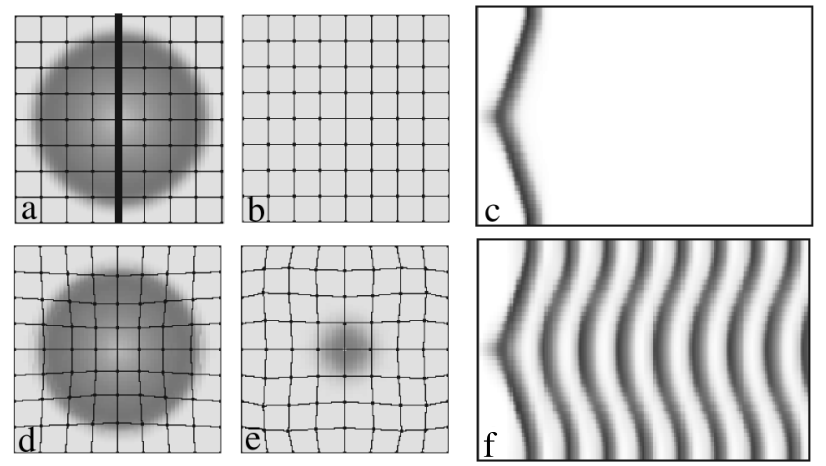

FIG. 1. Development of a stable pacemaker due to mechanical deformation. The upper panels show wave propagation in the absence of mechanical activity at time 10.17 time units (panel a), and 16.17 time units (panel b). Panel (c) shows the time-space plot for the bold vertical line marked in panel (a). The medium size was 28.8 space units and the total integration time spanning left-to-right in panel (c) is 100 time units. Panels (d)-(f) show a similar simulation to that in panels (a)-(c), but including mechanical deformation of the medium, with $G_{s}=0.5, E_{s}=1$, and $a=0.05$. The period of the pacemaker was 11.1 time units. For the time-space plots [panels (c) and (f)], time runs along the horizontal axis. pacemaking effect we observed. From Eq. (7), one can expect that the threshold value of the $I_{s}$ current necessary to initiate new excitations is proportional to $-G_{s}\left(u^{*}-E_{s}\right)$ (where $u^{*}$ is some constant). In order to test this, Fig. 3(a) illustrates the boundary of the onset of oscillations in the parametric space for $G_{s}^{-1}, E_{s}$. As expected, we see that $G_{s}^{-1}$ linearly increases with $E_{s}$. The relationship between $G_{s}$ and $a$ required for pacemaking was more complex [Fig. 3(b)]. We observed the onset of oscillations only for $a<0.085$. For $0.085 \leq a<0.12$ (above the dashed line), we obtained only one extra excitation that failed to propagate through the medium, and for $a \geq 0.12$ the propagation of the first wave also failed.

We found that if a pacemaker was established at any point other than the center of the medium, then it drifted to other locations in the medium. We performed computations by initiating the first pulse at 56 different locations throughout the medium. Almost all stimuli resulted in pacemakers that drifted to approach one of four attractors that were symmetrically located on the center lines across the medium, and at approximately $1 / 6$ of the medium size from the boundary, as illustrated in Fig. 4(a). In the cases where the stimulus was initiated on the diagonals of the medium, the pacemaker drifted to the central point, where it became stationary. The attractor locations were not affected by the size of mechanical elements. A $50 \%$ decrease in the element size resulted in negligible (less than $5 \%$ ) changes in the attractor locations. However, we found that the arrangement of attractor locations depended on the medium size [Fig. 4(b)]. If the medium size was decreased, then the peripheral attractors [open circles in Fig. 4(a)] shifted toward the boundaries of the medium. If the size was less than 25 space units, the pacemaking activity settled at the boundary of the medium [defined as shift $=$ 1 in Fig. 4(b)] at the middle of the corresponding side, similar to Fig. 4(a). For larger sizes, the four peripheral attractors approached the center of the medium. For media larger than 34 space units, a single attractor was located at the center of the medium ( sisted for all larger sizes of the medium for which computations were performed (up to 54 space units). In the limit of a very large medium (for which the relative influence of boundary is negligible), it is reasonable to expect that the pacemaker will either cease to drift or will drift indefinitely
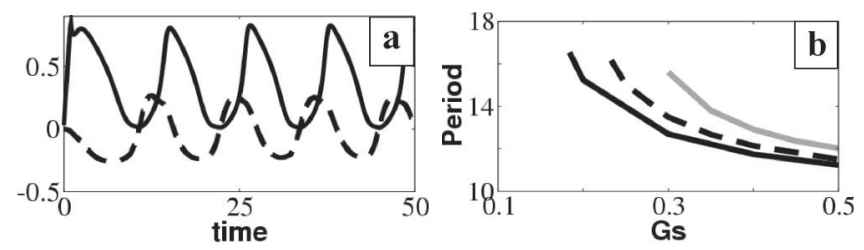

FIG. 2. (a) The time course of the excitation variable $u$ (solid line) and local dilatation $(\sqrt{C}-1)$ (dashed line) at center of the medium for the computation presented in Figs. 1(d)-1(f). (b) Period of the pacemaker as a function of $G_{s}$ for $a=0.05$ (solid line), $a=0.06$ (dashed line), and $a=0.07$ (gray line). 

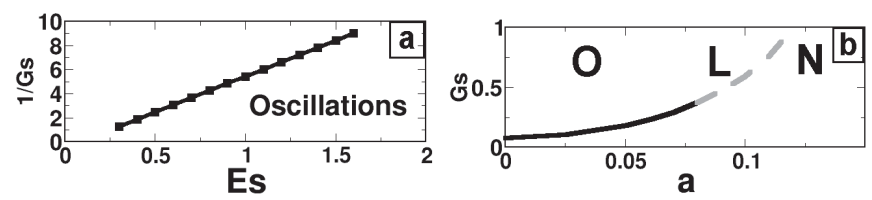

FIG. 3. Values of the parameters (a) $G_{s}^{-1}, E_{s}$, and (b) $G_{s}, a$, required for pacemaking oscillations. Symbols in panel (b) denote $\mathbf{O}$ : oscillations regime; $\mathbf{L}$ : single additional local response; and $\mathbf{N}$ : no wave propagation. Other parameter values were the same as those for Fig. 1.

(i.e., independent of a specific location). However, we were not able to approach this limit, nor find a substantial decrease in drift velocity towards the center for medium sizes larger than 34 space units. We found that pacemaker drift was due to a complex interaction between the depolarizing stretch-activated current and the recovery dynamics of the medium. For example, if the contraction was substantially delayed with respect to the excitation (more than 5 time units), then the pacemaker drift was always directed toward the boundary of the medium. We also found that the magnitude of the stretch-activated current at the point of initial stimulation was greater for a smaller medium compared to that for a larger medium. However, it remains to be determined how these observations can be used to robustly predict drift direction.

Finally, note that we were also able to initiate pacemaking activity using a single mechanical stimulus (i.e., a rapid local stretch) instead of electrical stimulation.

Discussion. - We found that mechanical deformation can induce pacemakers in excitable tissue, and that this effect is due to the depolarization action of the stretchactivated current. Although we use very general descriptions of the medium's excitation-mechanics properties and the dynamics of stretch-activated channels, we propose
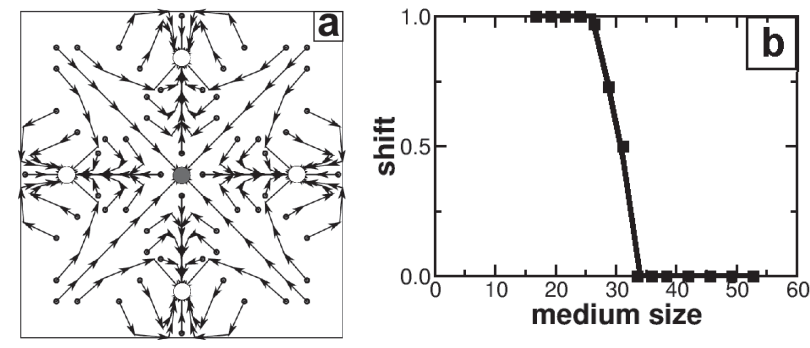

FIG. 4. Pacemaker drift. (a) Depending on the site of initiation, pacemakers drifted to one of five attractors symmetrically located throughout the medium - one central attractor (large filled circle) and four peripheral attractors (open circles). Computations were carried out using a medium of size $28.8 \times$ 28.8 space units; the size of each mechanical element was $7 \times 7$ electrical grid points. The arrows show the pacemaker trajectories, and filled black circles mark the initial sites of stimulation. (b) Relative shift-defined as the location of the peripheral attractors as a proportion of the distance from the center to the boundary of the medium-plotted against medium size. Computations were performed using $G_{s}=0.5, E_{s}=1$, and $a=0.05$. that this effect could be important in cardiac tissue. Indeed, as shown in detailed biophysical models of cardiac tissue [15], and in experimental studies [4], stretchactivated channels can depolarize cardiac tissue in a manner similar to that in our computations. The induction of a pacemaker depends on the relation of the depolarizing effect of $I_{s}$ with the excitation properties of cardiac cells. These properties differ substantially throughout the heart [4], and many types of cardiac cells show self-oscillating behavior, even in the absence of applied stretch. Therefore, given the wide variety of properties of cardiac cells and the depolarizing action of the stretch-activated channels in the heart, we propose that the effects of induction of pacemaking activity can exist for some types of cardiac cells, particularly those that exhibit self-oscillation dynamics.

One of limitations of our study is that our formulation neglects the fibrous anisotropy of cardiac tissue, which is important both for the electrical and the mechanical properties of the heart. We chose not to consider these effects, since the main aim of this study was to investigate the basic effects of deformation on a general reaction-diffusion system. The influence of cardiac anisotropy is likely to add additional effects, and will be addressed in future studies.

We are grateful to Professor P. J. Hunter, Dr. P. Kohl, and Professor H. Verschelde for valuable discussions. This research is funded by the Netherlands Organization for Scientific Research (NWO Grant No. 814.02.014).

[1] A. N. Zaikin and A.M. Zhabotinsky, Nature (London) 225, 535 (1970).

[2] C. Weijer, Curr. Opin. Genet. Dev. 14, 392 (2004).

[3] R. A. Gray and J. Jalife, Chaos 8, 65 (1998).

[4] P. Kohl, P. J. Hunter, and D. Noble, Prog. Biophys. Molec. Biol. 71, 91 (1999).

[5] R. Yoshida, T. Takahashi, T. Yamaguchi, and H. Ichijo, J. Am. Chem. Soc. 118, 5134 (1996).

[6] A. P. Muñuzuri et al., Phys. Rev. E 50, R667 (1994).

[7] V. N. Biktashev et al., Phys. Rev. Lett. 81, 2815 (1998).

[8] M. P. Nash and A. V. Panfilov, Prog. Biophys. Molec. Biol. 85, 501 (2004).

[9] V.S. Zykov, Simulation of Wave Processes in Excitable Media (Manchester University, Manchester, 1987).

[10] A. Garfinkel et al., Proc. Natl. Acad. Sci. U.S.A. 97, 6061 (2000).

[11] M. Stich, M. Ipsen, and A. S. Mikhailov, Phys. Rev. Lett. 86, 4406 (2001).

[12] A. S. Mikhailov et al., Proc. Natl. Acad. Sci. U.S.A. 101, 10890 (2004).

[13] L.E. Malvern, Introduction to the Mechanics of a Continuous Medium (Prentice-Hall, Englewood Cliffs, NJ, 1969).

[14] P. J. Hunter, M.P. Nash, and G. B. Sands, in Computational Biology of the Heart, edited by A. V. Panfilov and A. V. Holden (Wiley, Chichester, 1997), p. 345, Chap. 12.

[15] N. Trayanova, W. Li, J. Eason, and P. Kohl, Heart Rhythm 1, 67 (2004). 